\title{
Leaf Image Segmentation Based On the Combination of Wavelet Transform and K Means Clustering
}

\author{
N.Valliammal \\ Assistant Professor, Department of Computer Science, \\ Avinashilingam Institute of Home Science and Higher \\ Education for Women \\ Coimbatore, Tamilnadu, India.
}

\begin{abstract}
This paper focuses on Discrete Wavelet Transform (DWT) associated with the K means clustering for efficient plant leaf image segmentation. Segmentation is a basic pre-processing task in many image processing applications and essential to separate plant leafs from the background. Locating and segmenting plants from the background in an automated way is a common challenge in the analysis of plant images. Image segmentation is typically used to locate objects and boundaries (lines, curves, etc.) in images. Image segmentation is a fundamental task in agriculture computer graphics vision. Although many methods are proposed, it is still difficult to accurately segment an arbitrary image by one particular method. In recent years, more and more attention has been paid to combine segmentation algorithms and information from multiple feature spaces (e.g. color, texture, and pattern) in order to improve segmentation results .The performance of the segmentation is analyzed by Jaccard, dice, variation of index and global consistency error method. The proposed approach is verified with real time plant leaf data base. The results of proposed approach gives better convergence when compare to existing segmentation method.
\end{abstract}

Keywords- Image segmentation; Wavelet Transform; Haar Wavelet; K means clustering algorithm.

\section{INTRODUCTION}

Image segmentation is an essential step in many advanced techniques that covers multi-dimensional image processing and its applications. Digital Plant leaf analysis occupies an important place in many tasks such as in medical and agricultural field. Image segmentation techniques [6] are mostly used in plant leaf image processing field for detecting structures such as viens nodes, curvature and color of leaf detection. There are many approaches to image segmentation such as classifications, edges, or regions (Beaulieu and Touzi, 2004). Several techniques have been proposed for image segmentation using region growing (see Deng and Manjunath, 2001), graph cuts (see Boykov and Funka-Leam, 2006; Rother et al.2004), normalized cuts (see Shi and Malik, 2000), relaxation-based techniques (see Rosen-field et al., 1976), neural network based approaches (see Shan et al., 2005), methods based on fuzzy theory (see Hall, 1992), level sets (see Vese and Chan, 2002), and Markov random fields (see Schwartz and Pedrini, 2007). Comprehensive reviews of image segmentation techniques are described by $\mathrm{Pal}$ and $\mathrm{Pal}$ (1993) and Cremers (2007).

\author{
Dr.S.N.Geethalakshmi \\ Associate Professor, Department of Computer Science, \\ Avinashilingam Institute of Home Science and Higher \\ Education for Women \\ Coimbatore, Tamilnadu, India.
}

This paper describes the technique of wavelet transform use for features extraction associated with individual image pixels and combining this method with application of the $\mathrm{k}$ means clustering technique. For the image decomposition and feature extraction Haar transform has been applied as a basic tool used in the wavelet transform. A specific part of the paper is explained about the decomposition and reconstruction matrices. The method described is used for description of the whole system enabling perfect image reconstruction. The proposed algorithm of the Haar wavelet image decomposition includes image feature based segmentation with $\mathrm{k}$ means clustering algorithm. Individual methods have been verified for standard images and then applied for processing of selected real time plant leaf images.

In this paper, a proposed approach that makes use of wavelets and $\mathrm{K}$ means clustering is applied for leaf images. Through wavelets high pass image is extracted and to enhance edge details further decomposition is applied, which provides fine enhanced edge details with wavelet features like energy and entropy. The wavelet features and the $\mathrm{k}$ means are combined in our method to give better accuracy results.

The paper is organized as follows a brief literature survey is discussed in Section 2. The details of proposed approach is presented in Section 3 while Section 4 demonstrates the results of the proposed method. The conclusion is given in Section 5 .

\section{THE REVIEW OF WAVELET TRANSFORM}

The wavelet transform (WT), a linear integral transform that maps $L 2(\mathrm{R}) \rightarrow L 2\left(\mathrm{R}^{2}\right)$, has emerged over the last two decades as a powerful new theoretical framework for the analysis and decomposition of signals and images at multiresolutions [3].

Moreover, due to its locations in both time/space and in frequency, this transform is to completely differ from Fourier transform $[4,5]$. The region-based systems which use wavelet transform are classified into the following three categories according to the space units in which feature values for segmentation are calculated;

- A hierarchical block

- A moving window

- A pixel 
In [6], an image is segmented into hierarchical blocks by generating a quad tree. Then, for each block, statistics of wavelet coefficients (mean absolute value and variance) are computed in each subband, and used as features. The quad tree segmentation matches the multi level resolution analysis, and the features for each block can be obtained by applying wavelet transform only once to a whole image. Therefore, the computational cost is low. However, the method has low flexibility in the shapes of segmented regions. In [7], the method of nona-tree is proposed, instead of quad tree. Quad tree representation enables more flexible image segmentations. However, the computational cost is high, since the method requires wavelet transform for each block [21][22][23].

\section{A. Haar Wavelets in Image Decomposition}

Wavelets are functions generated from a single function by its dilations and translations. The Haar transform forms the simplest compression process of this kind. In 1-dimension, the corresponding algorithm [4] transforms a 2-element vector $[x(1), x(2)] T$ into $[y(1), y(2)] T$ by relation:

$$
\left[\begin{array}{l}
y(1) \\
y(2)
\end{array}\right]=\mathrm{T}\left[\begin{array}{l}
x(1) \\
x(2)
\end{array}\right] \quad \text { where } \mathrm{T}=\frac{1}{\sqrt{2}}\left[\begin{array}{cc}
1 & 1 \\
1 & -1
\end{array}\right]
$$

is an orthonormal matrix as its rows are orthogonal to each other (their dot products are zero). Therefore $\mathbf{T}-1=\mathbf{T} T$ and it is possible [4] to recover $\mathbf{x}$ from $\mathbf{y}$ by relation

$$
\left[\begin{array}{l}
x(1) \\
x(2)
\end{array}\right]=\mathbf{T}^{T}\left[\begin{array}{l}
y(1) \\
y(2)
\end{array}\right]
$$

In 2-dimensions $\mathbf{x}$ and $\mathbf{y}$ become $2 \times 2$ matrices. at first transform the columns of $\mathbf{x}$, by pre-multiplying by $\mathbf{T}$, and then the rows of the result by post-multiplying [4] by $\mathbf{T} T$ to find

$$
\mathbf{y}=\mathrm{TxT} T \text { and in the next step } \mathrm{x}=\mathrm{T} T \mathrm{yT}
$$

To show more clearly what is happening in a specific matrix is $\mathbf{x}$ of the form is applied

$$
x=\left[\begin{array}{ll}
a & b \\
c & d
\end{array}\right] \quad y=\frac{1}{\sqrt{2}}\left[\begin{array}{cc}
a+b+c+d & a-b+c-d \\
a+b-c-d & a-b-c+d
\end{array}\right]
$$

These operations correspond to the following filtering processes:

Top left: 2-D lowpass filter (Lo-Lo).

Top right: horizontal highpass and vertical lowpass filter

(Hi-L0)

Lower left: horizontal lowpass and vertical highpass filter

\section{(L0-Hi)}

Lower right: 2-D highpass filter (Hi-Hi).

To apply this transform to a complete image, the pixels are grouped into $2 \times 2$ blocks and apply Eq. (3) to each block. To view the result, all the top left components in of the $2 \times 2$ blocks in $\mathbf{y}$ were grouped together to form the top left subimage and the same for the components in the other three positions. It is clear from Fig. 1(b) that the most of the energy is contained in the top left (Lo-Lo) subimage and the least energy is in the lower right (Hi-Hi) subimage. The top right (Hi-Lo) and the lower left (Lo-Hi) subimage contains [4] the edges.

Following subsections describe algorithms of image segmentation using wavelet transform with resulting images presented in Fig. 1.

\section{B. Image Features Extraction}

Texture is characterized by the spatial distribution of gray levels in a neighborhood. An image region has a constant texture if a set of its local properties in that region is constant, slowly changing or approximately periodic. Texture analysis is one of the most important techniques used in analysis.

There are three primary issues in texture analysis: classification, segmentation and shape recovery from texture. Analysis of texture [1] requires the identification of proper attributes or features that differentiate the textures of the image. In this paper, texture segmentation is carried out by comparing co-occurrence matrix features. Contrast and Energy of size $N \times N$ is derived from discrete wavelet transform overlapping but adjacent subimages $\mathrm{Cij}$ of size $4 \times 4$ lays horizontally and vertically. The algorithm of image features extraction involves

1) Decomposition, using one level DWT with the Haar transform, of each subimage Ci,j of size $4 \times 4$ taken from the top left corner

2) Computation of the co-occurrence matrix features energy and contrast given in Eqs (3) and (4) from the detail coefficients, obtained from each subimage $C i, j$

\section{3) Forming new feature matrices}

\section{Circular Averaging Filtering}

In the image with the segmented band obtained after differences could appear with artifacts or spurious spots. Within the same region where the high differences of features values appear, the spots and noise were formed. These spurious elements were removed by applying a circular averaging filter. First the filter with suitable radius was created and then applied for a segmented image to minimize and denoise the image.

\section{Thresholding and Skeletonizing}

The processed image is then thresholded using global image threshold with Otsu's method [6] when a black and white image is obtained. The appearance of thick boundaries has to be thinned on the line for one pixel thickness. To process these specific morphology operations were used.

At first operation 'clean' removes isolated pixels individual 1's that are surrounded by 0 's.

The second operation 'skel' removes pixels on the boundaries of objects but does not allow objects to break apart. The remaining pixels make up the image skeleton. 


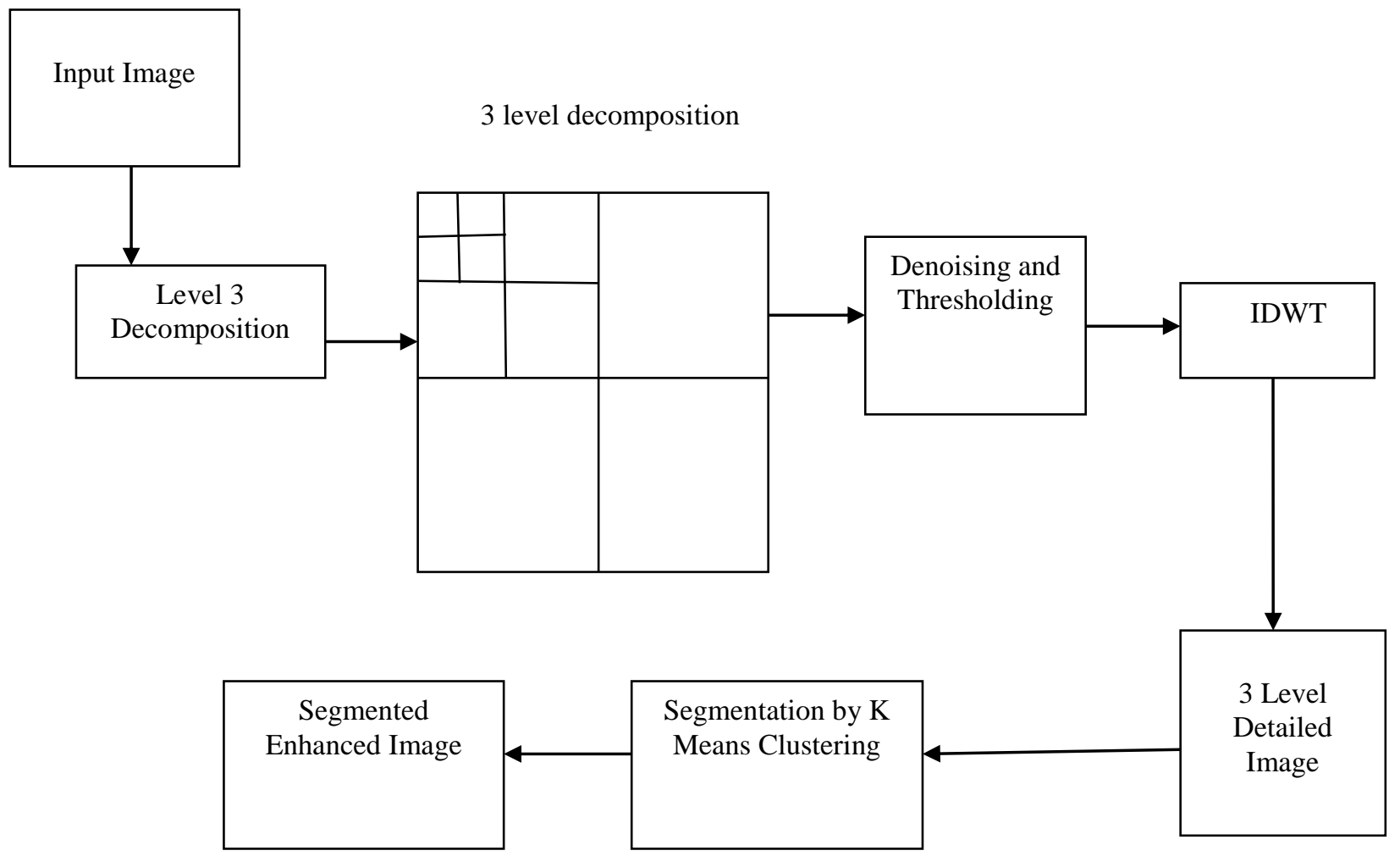

Figure 1 Proposed Methodology for Plant Leaf Image Segmentation

\section{K MEANS CLUSTERING}

Clustering is a process of organizing the objects into groups based on its attributes. A cluster is therefore a collection of objects which are "similar" between them and are "dissimilar" to the objects belonging to other clusters. An image can be grouped based on keyword (metadata) or its content (description).

In keyword based clustering, a keyword is a form of font which describes about the image keyword of an image refers to its different features. The similar featured images are grouped to form a cluster by assigning value to each feature.

In content based clustering [10], [11], [20] , [24],[25] a content refers to shapes, textures or any other information that can be inherited from the image itself. The tools, techniques and algorithms that are used originate from fields such as statistics, pattern recognition, signal processing etc. Clustering based on the optimization of an overall measure is a fundamental approach explored since the early days of pattern recognition.

The most popular method used efficiently for pattern recognition is K-means clustering.

In K-means clustering a centroid vector is computed for every cluster. The centroid must be chosen such that it should minimize the total distance within the clusters.

Both supervised and unsupervised clustering techniques are used in image segmentation. In supervised clustering method, grouping is done according to user feedback. In unsupervised clustering, the images with high features may be very different in terms of semantics [16].

In K-means algorithm data vectors are grouped into predefined number of clusters [12][13]. At the beginning the centroids of the predefined clusters are initialized randomly. The dimensions of the centroids are same as the dimension of the data vectors. Each pixel is assigned to the cluster based on the closeness of the pixel [14], which is determined by the Euclidian distance measure. After all the pixels are clustered, the mean of each cluster is recalculated. This process is repeated until no significant changes result for each cluster mean or for some fixed number of iterations.

\section{A. Global K-Means Algorithm}

According to Likas [8] the global k-means clustering algorithm does not depend upon the initial parameter values and utilize the k-means algorithm as a local search procedure that constitutes a deterministic global optimization method. This technique proceed in an incremental way of attempting to optimally include one new cluster center at each stage instead of randomly selecting starting value for all clusters [17][18]. More particularly, to solve a clustering problem with k clusters the method proceeds as follows.

Step 1: The algorithm begins with one cluster $(\mathrm{k}=1)$ and cluster center corresponds to the centroid of the data set $\mathrm{X}$.

Step 2: Perform $\mathrm{N}$ executions of the $\mathrm{k}$-means to find two cluster $(\mathrm{k}=2)$ after the initial positions of the cluster centers: For $\mathrm{k}=1$, the first cluster center is constantly placed at the optimal position. The second center at execution $n$ is placed at 
the position of the data point $\mathrm{xn}(\mathrm{n}-1, \ldots \ldots, \mathrm{N})$. The best solution is obtained after $\mathrm{N}$ executions of the k-means algorithm.

Step 3: For k-1 clustering problem, the final solution is denoted by $(\mathrm{c} 1, \mathrm{c} 2, \ldots, \mathrm{ck}-1)$.

The algorithm performs $\mathrm{N}$ execution of the k-means algorithm with initial positions $(\mathrm{c} 1, \mathrm{c} 2 \ldots \ldots$. ck-1, xn), where $\mathrm{n}$ varies from 1 to $\mathrm{N}$ to find solution for $\mathrm{k}$-clustering problem. The best solution obtained in this process is considered as the final solution.

\section{PROPOSED Methodology}

Our proposed methodology is a five-step process, graphically illustrated in Fig. 1. All these steps are briefly depicted below:

1. Wavelet transform is applied to an input Plant Leaf image to obtain wavelet decomposed image resulting in twelve subbands. All these subbands represent approximation, horizontal, vertical and diagonal components in the form of coefficients, respectively. LL subband contains low level and the other three ( $\mathrm{LH}, \mathrm{HL}, \mathrm{HH})$ contain high level details.

2. Denoise the decomposed leaf image using average filter and Global thresholding .Otsu's model is applied for separating the background and the shape of leaf is extracted.

3. Set approximation coefficients in $\mathrm{LL}$ equal to zero and apply inverse wavelet transform to obtain a high pass image from the remaining (horizontal, vertical and diagonal) subbands. We call the resultant image level-3 (L3) detail image.

4. Add L3 to the original image to get a sharpened image.

5. Apply the $\mathrm{K}$ means clustering algorithm, as given in step [3], to segment the images that partition the data set into an optimal number of clusters. Same data points belong to one cluster and different data points belong to different clusters.

This algorithm accounts for variability in cluster shapes, cluster densities and the number of data points in each of the subsets. This $\mathrm{K}$ means has a drawback that it cannot work well in case of noisy plant leaf images. Therefore, the combination of wavelet and $\mathrm{K}$ means provides better results as wavelets are robust to noise and this combination also helped to remove in homogeneity and artifacts produced in plant leaf imaging

6. The edge information is rectified using sobel edge detector and finally the enhanced image is obtained.

Discrete Wavelet Transform (DWT) is applied to plant leaf images because wavelets provide frequency information as well as time-space localization. In addition, their multiresolution character enables to visualize image at various scales and orientations. The multi-resolution property provides information about various high frequency components at different levels of decomposition.

Over-decomposition should however be avoided, because as the decomposition levels increase, there is a great risk that lower frequencies become a part of detail components. This may restrict us to use only fewer level of decomposition because lower frequencies will become part of high pass image and reduce effective detail in an image.

\section{EXPERIMEBNTAL RESULTS AND ANALYSIS}

The proposed approach to a large set of Plant Leaf images forms a standard database. The step-by-step process, after applying the proposed method, is shown in Fig. 3. The performance of the proposed segmented algorithm is checked with FCM segmentation algorithm using clustering performance parameter jaccard, dice, variation of index and global consistency error method.

The input image is decomposed by DWT at level-3 and it gives a detail image (Fig.b) by setting subband to zero, applying the average filter for denoising noisy pixel image and to extract the object global thresholding and thereafter applying inverse DWT. The resultant detail image is then added to the original input image to result in a sharpened image shown in (Fig. e).

For Level 3 decomposed image $\mathrm{K}$ means algorithm is applied as shown in (Fig. d). It can be seen that contours are not properly highlighted and edge information is missing in some places.

To preserve edge information, sobel edge detection mask is applied to fill edges in cluster segmented image and the result is shown in (Fig. f).

\section{A. Jaccard}

The Jaccard index, also known as the Jaccard similarity coefficient is a statistic used for comparing the similarity and diversity of sample sets. The Jaccard coefficient measures similarity between sample sets, and is defined as the size of the intersection divided by the size of the union of the sample sets:

$$
J(A, B)=\frac{|A \cap B|}{|A \cup B|} .
$$

\section{B. Dice}

Dice coefficient, is a similarity measured over sets:

$$
s=\frac{2|X \cap Y|}{|X|+|Y|}
$$

It is identical to the Sørensen similarity index, and is occasionally referred to as the Sørensen-Dice coefficient.

\section{Variation of Information (VOI)}

The Variation of Information (VOI) metric defines the distance between two segmentations as the average conditional entropy of one segmentation given to the other, measures the amount of randomness in one segmentation which cannot be explained by the other [21]. Suppose there are two clustering (a division of a set into several subsets) $\mathrm{X}$ and $\mathrm{Y}$ where $\mathrm{X}=\{\mathrm{X} 1, \mathrm{X} 2 \ldots \mathrm{Xk}\}, \mathrm{pi}=|\mathrm{Xi}| / \mathrm{n}, \mathrm{n}=\Sigma \mathrm{k}|\mathrm{Xi}|$. Then the variation of information between two clustering is:

$$
\mathrm{VI}(\mathrm{X} ; \mathrm{Y})=\mathrm{H}(\mathrm{X})+\mathrm{H}(\mathrm{Y})-2 \mathrm{I}(\mathrm{X}, \mathrm{Y})
$$

Where, $\mathrm{H}(\mathrm{X})$ is entropy of $\mathrm{X}$ and $\mathrm{I}(\mathrm{X}, \mathrm{Y})$ is mutual information between $\mathrm{X}$ and $\mathrm{Y}$. The mutual information of two clustering is the loss of uncertainty of one clustering if the 
other is given. Thus, mutual information is positive and bounded by $\{\mathrm{H}(\mathrm{X}), \mathrm{H}(\mathrm{Y})\}_{-} \log 2(\mathrm{n})$.

\section{Global Consistency Error (GCE)}

The Global Consistency Error (GCE) measures the extent to which one segmentation can be viewed as a refinement of the other [21]. Segmentations which are related are considered to be consistent, could represent the same image segmented at different scales. Segmentation is simply a division of the pixels of an image into sets. The segments are sets of pixels. If one segment is a proper subset of the other, then the pixel lies in an area of refinement, and the error should be zero. If there is no subset relationship, then the two regions overlap in an consistent manner. The formula for GCE is as follows:
Where, segmentation error measure takes two segmentations S1 and S2 as input, and produces a real valued output in the range [0::1] where zero signifies no error. For a given pixel pi consider the segments in $\mathrm{S} 1$ and $\mathrm{S} 2$ that contain that pixel.

The figure 3 shows the image results for different stages in proposed methodology. The proposed methodology consists of five stages. The input image, wavelet level 3 decomposed image, denoising and thresholding and finally enhanced segmentation image is shown in figure 2 . In table 1 , the proposed methodology gives the suitable results on the basis of comparing the jaccard, dice, variation of index and global consistency error.

$\mathrm{GCE}=1 / \mathrm{n} \cdot \min \mathrm{E}(\mathrm{s} 1, \mathrm{~s} 2, \mathrm{pi}), \mathrm{E}(\mathrm{s} 2, \mathrm{~s} 1, \mathrm{pi})$

TABLE 1 PARAmEtric EVAluation For DifFERENT SEgMENTATION ALGORITHM

\begin{tabular}{|l|c|c|c|c|}
\hline Algorithm & Jaccard & Dice & VOI & GCE \\
\hline FCM & 0.9455 & 0.0167 & 0.872 & 0.563 \\
\hline Wavelet & 0.9440 & 0.0113 & 0.631 & 0.452 \\
\hline K means & 0.953 & 0.0142 & 0.825 & 0.713 \\
\hline Proposed method & 0.9688 & 0.0091 & 0.544 & 0.412 \\
\hline
\end{tabular}

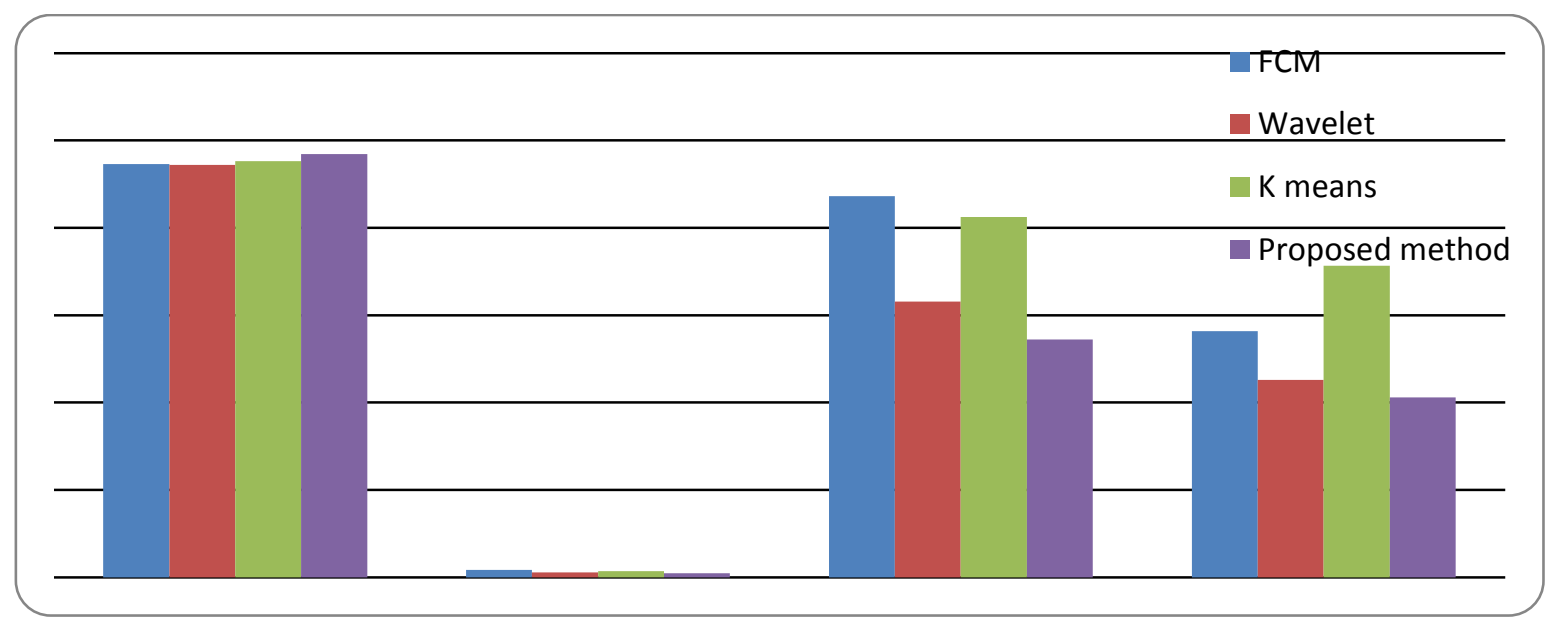

Figure- 2 Graphical results for Different Segmentation Algorithm 


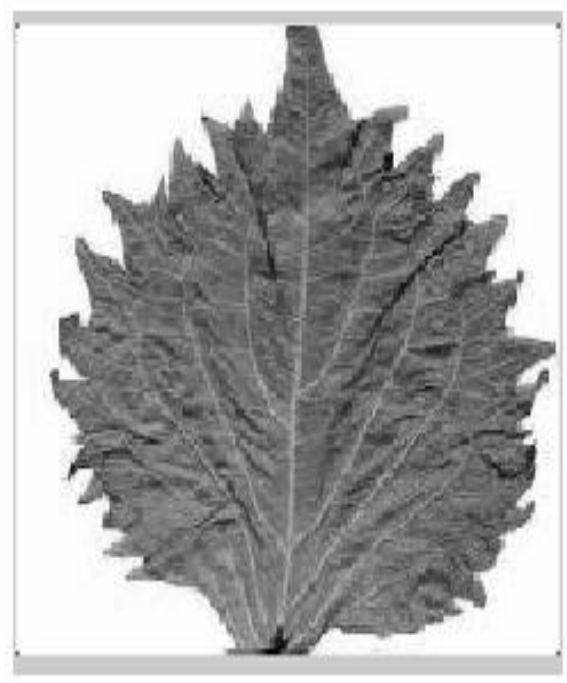

Fig-a- Original Image

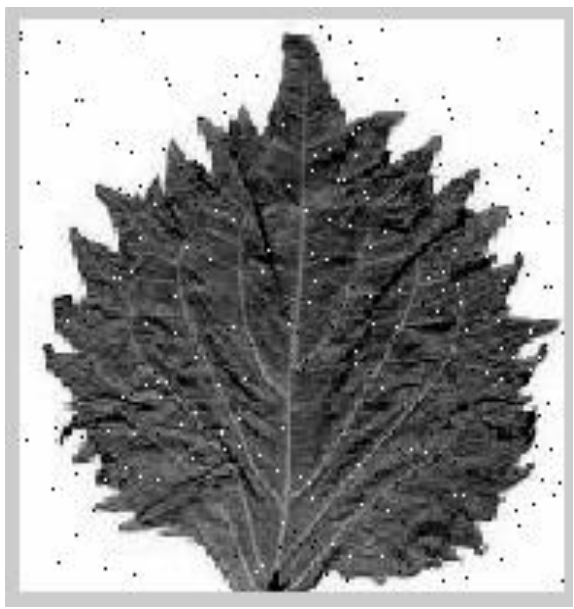

Fig-c- Noisy Pixel Image

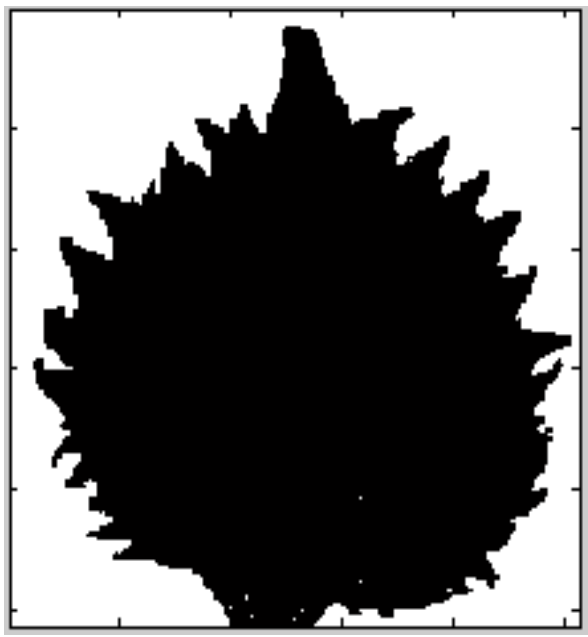

Fig-e- Global Thresholding

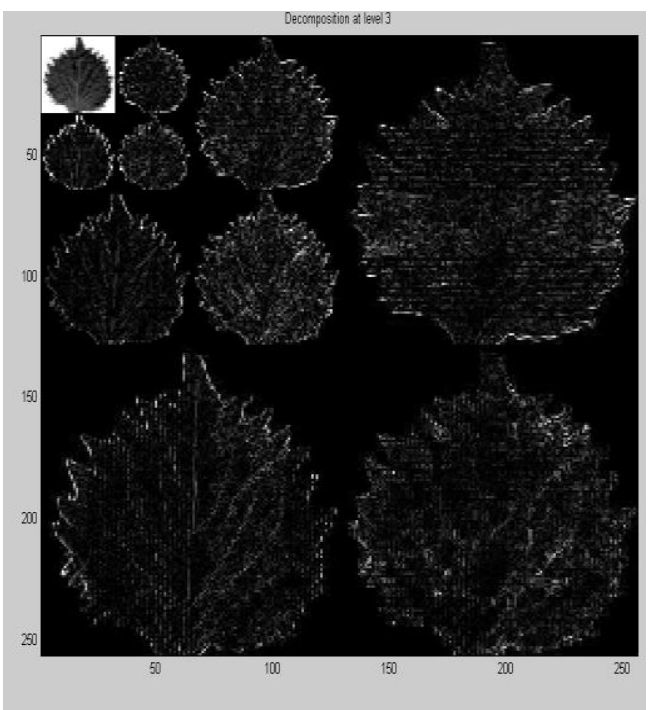

Fig-b- 3 Level Decomposed Image

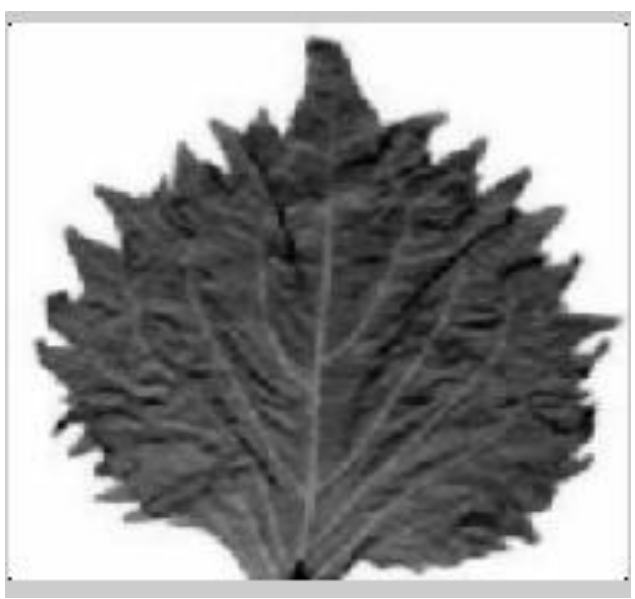

Fig-d-Denoised by Average Filter

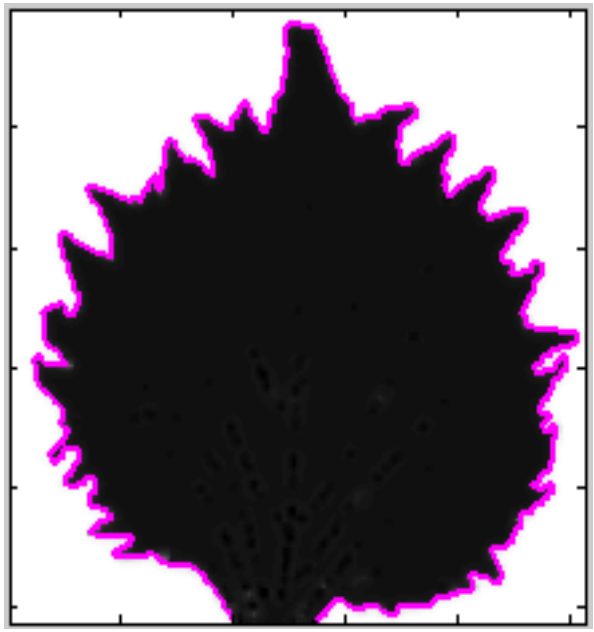

Fig-f- K means and Sobel Enhanced Image

Figure- 3 Image results for Different Stage in Proposed Methodology 


\section{CONCLUSION}

This paper discussed on Discrete Wavelet Transform (DWT) associated with the $\mathrm{K}$ means clustering for efficient plant leaf image segmentation. The performance of the segmentation is analyzed by Jaccard, dice, variation of index and global consistency error method.

The proposed approach is verified with real time plant leaf data base. The results of the proposed approach give better convergence when compared to conventional segmentation method.

\section{REFERENCE}

[1] Juin-Der Lee, Hong-Ren Su, MR Image Segmentation Using a Power Transformation Approach, P.1-13, 2008.

[2] C. H. Chen, G. G. Lee, Image Segmentation Using Multiresolution Wavelet Analysis and Expectation-Maximization (EM) Algorithm for Digital Mammography,wiley, 1997.

[3] Neelum Noreen, Khizar Hayat and Sajjad A. Madani, MRI Segmentation through Wavelets and Fuzzy C-Means, World Applied Sciences Journal 13 (Special Issue of Applied Math): p. 34-39, 2011.

[4] R. C. Dubes and A. K. Jain. Algorithms for Clustering Data.Prentice Hall, 1988.

[5] T. Zhang, R. Ramakrishnan, and M. Livny. BIRCH: An Efficient Data Clustering Method for very Large Databases. Proc. of the 1996 ACM SIGMOD Int'l Conf. on Management of Data, Montreal, Canada, pages 103-114, June 1996.

[6] 7. S. Thilagamani1 and N. Shanthi2, A Survey on Image Segmentation Through Clustering, International Journal of Research and Reviews in Information Sciences Vol. 1, No. 1, P.14-18March 2011.

[7] G.C. Tseng. Penalized and weighted k-means for clustering with scattered objects and prior information in high-througput biological data. Bioinformatics, Vol.23, No.17, P.2247-2255, 2007.

[8] Mrunalini R. Badnakhe and Prashant R. Deshmukh, An Application of K-Means Clustering and Artificial Intelligence in Pattern Recognition for Crop Diseases, proceeding of International Conference on Advancements in Information Technology With workshop of ICBMG 2011, P.134-139,2011.

[9] R.Srinivasa Perumal, R.Sujatha, Analysis of Colon Cancer Dataset using K-Means based

[10] Algorithms \& See5 Algorithms, IJCST Vol. 2, No. 4, P.482-485, 2011.
[11] B.Sathya, R.Manavalan, Image Segmentation by Clustering Methods: Performance Analysis, International Journal of Computer Applications Volume 29- No.11, P.0975 - 8887, 2011.

[12] 12. M. Sesli1* and E. D. Yegenoglu2, Compare various combinations of similarity coefficients and clustering methods for Olea europaea sativa, Scientific Research and Essays Vol. 5, No.16, P. 2318-2326, 2010.

[13] 13. Byung-Gyu Kim, Jae-Ick Shim, Dong-Jo Park, Fast image segmentation based on multi- esolution analysis and wavelets, Pattern Recognition Letters 24, P. 2995-3006, 2003.

[14] 14. J J . Lewis, R J O Callaghan, S G Nikolov, D R Bull, Region-Based Image Fusion Using Complex Wavelets, Image Rochester NY, Vol. 8, No. 2, Publisher: Citeseer, Pages: 119-130,2007.

[15] 15. Gonzalez, R. C.; Woods, R. E. 2002. Digital Image Processing. Prentice Hall, Inc., Upper Saddle River, New Jersey.

[16] 16. Lj.Jovanov, A. Pi`zurica, and W. Philips, "Wavelet based joint denoising of depth and luminance images," in 3D TV conference, (Kos Island, Greece), 2007.

[17] 17. P. Scheunders, "Wavelet thresholding of multivalued images," IEEE Trans. Image Proc. 13, pp. 475- 483, 2004.

[18] 18. P. Scheunders and S. De Backer, "Wavelet denoising of multicomponent images, using a noise-free image," in Proc. IEEE Internat. Conf. Image Proc. ICIP, 2006.

[19] 19. P. Jayamala K. Patil, Raj Kumar, Advances in Image Processing for Detection of plant diseases, Journal of Advanced Bioinformatics Applications and Research ISSN 0976-2604 Vol 2, N0. 2, June-2011. pp 135-141.

[20] zGurbinder kaur, Intensity based image Segmentation using Wavelet analysis and Clustering techniques, indian journal of computer science and engineering (ijcse), vol. 2 no. 3 jun-jul 20

[21] Mr. Viraj A. Gulhane, Dr. Ajay A. Gurjar, Detection of Diseases on Cotton Leaves and Its PossibleDiagnosisInternational Journal of Image Processing (IJIP),Vol.5 , No.5, P.590-598, 2011.

[22] Nourhan M. Zayed (et.al), Wavelet Segmentation for Fetal Ultrasound Images, IEEE paper, 2010.

[23] Yui-Liang Chen (et.al), Color Image Segmentation Using Wavelet Transform Techniques, 16th IPPR Conference on ComputerVision, Graphics and Image Processing (CVGIP 2003), P.669-675, 2003.

[24] Gurpreet kaur and Himanshu Monga, Classification of Biological Species Based on Leaf Architecture-A review, IRACST - International Journal of Computer Science and Information Technology \& Security (IJCSITS),Vol. 2, No.2, P.332-334, 2012.

[25] Jyotismita Chaki and Ranjan Parekh, Plant Leaf Recognition using Shape based Features and Neural Network classifiers, (IJACSA) International Journal of Advanced Computer Science and Applications, Vol. 2, No. 10, P.41-48,2011 